Article

\title{
A Single-Cell Study of a Highly Effective Hog1 Inhibitor for in Situ Yeast Cell Manipulation
}

\author{
Charlotte Hamngren Blomqvist ${ }^{1,2}$, Peter Dinér ${ }^{3,4}$, Morten Grøtli ${ }^{4}$, Mattias Goksör ${ }^{1}$ \\ and Caroline B. Adiels ${ }^{1, *}$
}

1 Department of Physics, University of Gothenburg, Göteborg S-412 96, Sweden;

E-Mails: charlotte.hamngren.blomqvist@ chalmers.se (C.H.B.);

mattias.goksor@physics.gu.se (M.G.)

2 Department of Applied Physics, Chalmers University of Technology, Göteborg S-412 96, Sweden

3 Department of Chemistry, KTH-Royal Institute of Technology, Teknikringen 30, SE-10044

Stockholm, Sweden; E-Mail: diner@kth.se

4 Department of Chemistry and Molecular Biology, University of Gothenburg,

Göteborg S-413 90, Sweden; E-Mail: grotli@ chem.gu.se

* Author to whom correspondence should be addressed; E-Mail: caroline.adiels@physics.gu.se;

Tel.: +46-31-786-9123.

Received: 11 November 2013; in revised form: 20 January 2014 / Accepted: 6 February 2014 /

Published: 19 February 2014

\begin{abstract}
We present a single cell study of a highly effective Hog1 inhibitor. For this application, we used sequential treatment of a Saccharomyces cerevisiae cell array, with the Hog1 inhibitor and osmotic stress. For this purpose, a four-inlet microfluidic chamber with controlled introduction of two different cell strains within the same experimental setting and a subsequent rapid switching between treatments was designed. Multiple cell strains within the same experiment is a unique feature which is necessary for determining the expected absent cellular response. The nuclear translocation of the cytosolic MAPK, Hog1, was monitored by fluorescence imaging of Hog1-GFP on a single-cell level. An optical tweezers setup was used for controlled cell capture and array formation. Nuclear Hog1-GFP localization was impaired for treated cells, providing evidence of a congenial microfluidic setup, where the control cells within the experiments validated its appropriateness. The chamber enables multiple treatments with incubation times in the order of seconds and the possibility to remove either of the treatments during measurement. This flexibility and the possibility to use internal control cells ensures it a valuable scientific tool for unraveling the HOG pathway, similar signal transduction pathways
\end{abstract}


and other biological mechanisms where temporal resolution and real time imaging is a prerequisite.

Keywords: microfluidics; single-cell analysis; MAPK; inhibitor; optical trapping; cell-to-cell variability; system biology; Saccharomyces cerevisiae; HOG; optical tweezers

\section{Introduction}

The emerging interest for single-cell analytical data calls for novel data acquiring methods. Microfluidics can effectively be used in studies of signal transduction pathways by the fast and reproducible introduction of different perturbations on the studied system. Single-cell analysis of yeast was first reported in the early 50's [1], and includes several different single-cell assays [2]. The spatial and temporal resolutions of such analyses are widely exceeding that of traditional cell culture methodologies [3]. In addition, single-cell analysis provides information on cell-to-cell variability that will be obscured in averaged results from population analyses [4]. This heterogeneity in cell response can be attributed to intrinsic and extrinsic noise [5], e.g., in which phase the individual cells are in their cell cycle. Single-cell analyses have been improved further by the use of microfluidics. The microfluidic chambers are cheap to produce, customizable and compatible with different spectrometric methods and, in addition, they also enable the possibility of accomplishing fast and accurate concentration changes of, e.g., nutrients and different substances that affect the biological event studied. In order to control not only the microenvironment around the cells in the microfluidic chamber, but also the cell positions, optical tweezers (OT) are used [6]. This enables selective cell manipulation in a non-intrusive way [7].

The study of biological signaling pathways is complicated, and Saccharomyces cerevisiae (S. cerevisiae), also known as budding yeast due to its reproduction mechanism, is a frequently used eukaryote model organism [8-10] for studying biological mechanisms that could have future impact on human disease prevention [11]. The High Osmolarity Glycerol (HOG) Mitogen Activated Protein Kinase (MAPK) pathway of $S$. cerevisiae, a functional homolog of the stress activated MAPK signaling pathway Jun $\mathrm{N}$-terminal kinase (JNK) and the MAPK p38 pathways of mammals [12], is involved in the cellular adaptation to hyperosmotic stress. Because of the high degree of conservation of the MAPK kinase cascades, the yeast HOG pathway is a good model for studying osmotic adaptation processes. The HOG pathway responds to changes in external osmolarity by activating the MAPK Hog1, triggering the cellular response that includes both transcriptional upregulation of approximately six hundred osmoresponsive genes [13-15], and events extending beyond gene transcription. The closure of the glycerol export channels in the plasma membrane [16] and rerouting of the metabolite utilization in glycolysis [17] will contribute to an increased cellular glycerol content and cell adaptation to the new environment. Three transmembrane proteins, Sln1, Msb2 and Hkr1 sense the increased external osmolarity $[18,19]$. The activation signal is transduced via two branches (the SIn1 and Sho1 branch respectively) that merge by the phosphorylation of the MAPK kinase (MAPKK) Pbs2. Pbs2 subsequently phosphorylates and activates the MAPK Hog1, which rapidly translocates from the cytoplasm into the nucleus [20] via the nuclear envelope transport protein, 
Nmd5p [21]. A simplified schematic of the HOG signaling pathway can be seen in Figure 1. The phosphorylation of Hogl is required for its nuclear envelope translocation [21]. The influence of the used Hog1 inhibitor on the translocation of Hog1 has been described by Dinér et al. [22]. Migration studies upon Hog1 pathway activation, where the intensity ratio of nucleic to cytosolic Hog1-GFP is plotted, would reveal if the effects of Hog1 inhibition (i.e., a prolonged phosphorylation and hence nuclear localization of $\operatorname{Hog} 1)$ were mirrored in the protein's location.

Figure 1. A simplified scheme of the HOG signaling pathway. The HOG pathway responds to changes in external osmolarity. The activation signal is transduced via the SIn 1 and Sho1 branch respectively, that merge by the phosphorylation of the MAPKK Pbs2. Pbs2 subsequently phosphorylates and activates the MAPK Hog1, which rapidly translocates from the cytoplasm into the nucleus. The inhibitor acts on Hog 1 and will inhibit its downstream kinase activity.

Increased extracellular

osmotic pressure

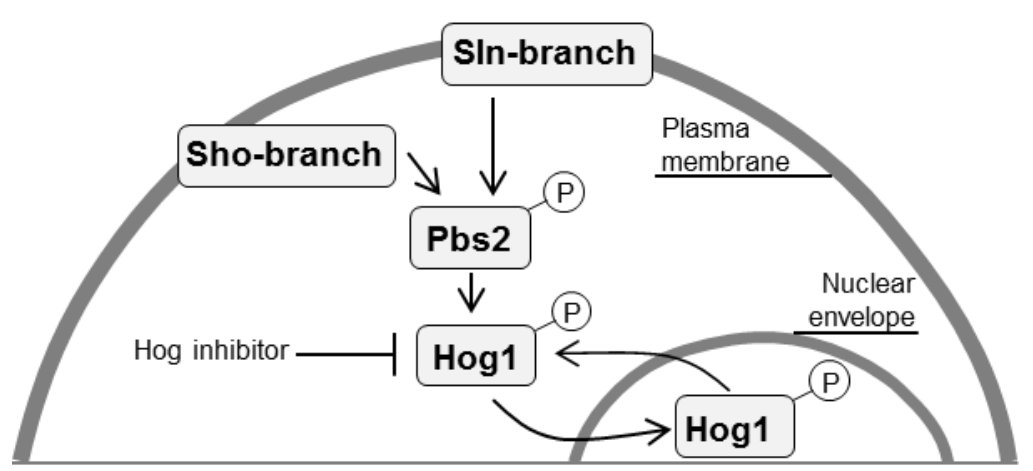

In this article, we report on a single-cell evaluation of the highly effective Hog 1 inhibitor 4-(1-benzyl-4-phenyl-1H-1,2,3-triazol-5-yl)- $N$-isopropylpyridin-2-amine [22] for the HOG pathway. A four-inlet microfluidic chamber, enabling rapid switching between different treatments, was utilized for analyzing the stress-induced translocation process of Hog 1 as a function of time. Cell responses due to osmotic stress after and during exposure with a Hog1-specific inhibitor was studied using two distinguishable cell strains within the same chamber. Sequential Hog1 inhibitor and osmotic stress treatment of an S. cerevisiae cell array were performed, with temporal resolution and an experimental precision down to a few seconds. This temporal precision was essential in order to determine the most efficient incubation time for Hog 1 inhibition and to have comparable measurements with a minimized temporal variation of incubation times between the different single-cell experiments.

Our setup enables actively choosing single cells from two different cell strains and following the responses of individual cells over time. This possibility makes the setup advantageous for unraveling the mechanisms of the HOG and similar dynamic signal transduction pathways. Any heterogeneous response of the single cells is revealed by using the intensity ratio of fluorescently tagged proteins within the cell as a tool for tracing protein movement. For the possible event of complete inhibition, a method to ensure that cells were responding to the inflowing chemical as intended had to be 
established. The solution was to introduce a second cell strain using the additional inlet of the four-inlet chamber. These control cells were imaged alongside the cells of interest when the Hog1 inhibitor was used. This increase in versatility and the use of in situ control cells ensures our chamber a valuable tool for providing insight into biological problems, e.g., the complexity of the signaling pathways of $S$. cerevisiae.

A key improvement over our previously described three-inlet chamber [7], is that the four-inlet microfluidic chamber allows for rapid switching between two treatments, which leads to a great gain in temporal resolution.

\section{Experimental Section}

The cells were captured and positioned using OT (400 mW Ytterbium fibre laser LP, $1070 \mathrm{~nm}$, IPG Laser) and were sequentially treated with a selective Hog1 kinase inhibitor [22] and sorbitol. These single-cell experiments are the first using this particular Hog1 inhibitor. Responses were monitored on a single-cell level by time-lapse fluorescence microscopy, monitoring the nuclear migration of the reporter protein Hog1-GFP, initiated by osmotic stress. The flow of Hog1 inhibitor was introduced to the cells via one of the four inlets. Two other inlets carried the two cell strains respectively, and the final one introduced the flow of sorbitol (for increased osmotic pressure upon the cells). The microscope was a Leica DMI6000B with control box CTR 6500 (Leica Microsystems, Wetzlar, Germany). The GFP filter cube was a 472/30 nm exciter, 520/35 nm emitter and 495LP dichroic mirror (Semrock IDEX Corporation, Rochester, NY, USA). The mCherry filter cube was a 560/40 nm exciter, 630/75 nm emitter and 585LP dichroic mirror (Chroma, Bellows Falls, VT, USA). A 100× HCX plan fluotar oil immersion objective (Leica Microsystems, Wetzlar, Germany) with a numerical aperture of 1.30 was used.

\subsection{Strains and Cell Culture}

Two different yeast strains in BY4741 background were used; the HOG1-GFP-HIS3 NRD1-mCherry-hphNT1 strain, for monitoring Hog1 migration, and the MSN2-GFP-HIS3 NRD1-mCherry-hphNT1 strain as control cells. Both strains were grown in Yeast Nitrogen Base (YNB, $6.7 \mathrm{~g} / \mathrm{L})$ with Complete Supplement Mixture (CSM, $1.54 \mathrm{~g} / \mathrm{L})$ and $2 \%$ Glucose $(\mathrm{pH}=6)$, at $30{ }^{\circ} \mathrm{C}$ on a shaker $(220 \mathrm{rpm})$. The two cell strains were collected at $\mathrm{OD}_{600}=0.5-1.0$. The Hog1-GFP cells were then concentrated to twice the initial concentration by centrifugation at a relative centrifugal force of $2400 \times g$ for $30 \mathrm{~s}$, followed by removal of the supernatant and addition of fresh growth medium. The cell density of the Hog1-GFP cells were 3 to 4 times the cell density of the Msn2-GFP cells.

\subsection{Inhibitor}

The selective Hog1 inhibitor 4-(1-benzyl-4-phenyl-1H-1,2,3-triazol-5-yl)- $N$-isopropylpyridin-2amine [22] ( $\mathrm{M}=478.67 \mathrm{~g} / \mathrm{mol}$ ) (see Figure $\mathrm{S} 1$ for chemical structure) was dissolved in dimethyl sulfoxide (DMSO) to a suitable stock solution. Final concentrations range between $10 \mathrm{nM}$ and $25 \mu \mathrm{M}$ and control experiments contained the corresponding maximum volume of DMSO. The approximated diffusion coefficient was determined to $260 \mu \mathrm{m}^{2} / \mathrm{s}$ (measured at $22^{\circ} \mathrm{C}$ ). 


\subsection{Microfluidic Fabrication and Setup}

The manufacturing protocol for the microfluidic chamber and the experimental procedure has previously been reported [7,23,24]. The disposable microfluidic chambers were made from polydimethylsiloxane (PDMS) using soft lithography and sealed off with a cover glass. A reusable master relief pattern of $27 \mu \mathrm{m}$ height was formed on a silicon wafer and PDMS was poured onto it using a formwork holding pins to form the four inlet holes and the outlet of the microfluidic chamber. After polymerization, the PDMS structure and a glass slide were treated with air plasma and thereafter immediately placed in physical contact with each other, spontaneously forming a strong irreversible seal [25]. Figure 2 shows a schematic image of the four-inlet microfluidic chamber and workflow setup, and a colored overlay where both the cells of interest and the control cells are shown.

Figure 2. Schematic image of the setup in relation to the workflow. (A) The syringes filled with cells and substances are placed in a syringe pump. Cells are trapped by OT and positioned in an array within the microfluidic chamber. The cells and their reporter proteins are imaged in transmission light and fluorescent light while the pump flow rates are automatically controlled and altered; (B) a colored overlay of the bright field, GFP and mCherry images. Cells were actively placed in an array using the OT. In the four first columns, cells are expressing Hog1-GFP and in the rightmost column, control cells are instead expressing Msn2-GFP. The two cell types are visually distinguishable by the different GFP expression. Both cell types express the nucleic protein Nrd1-mCherry. The scale bar is $10 \mu \mathrm{m}$.
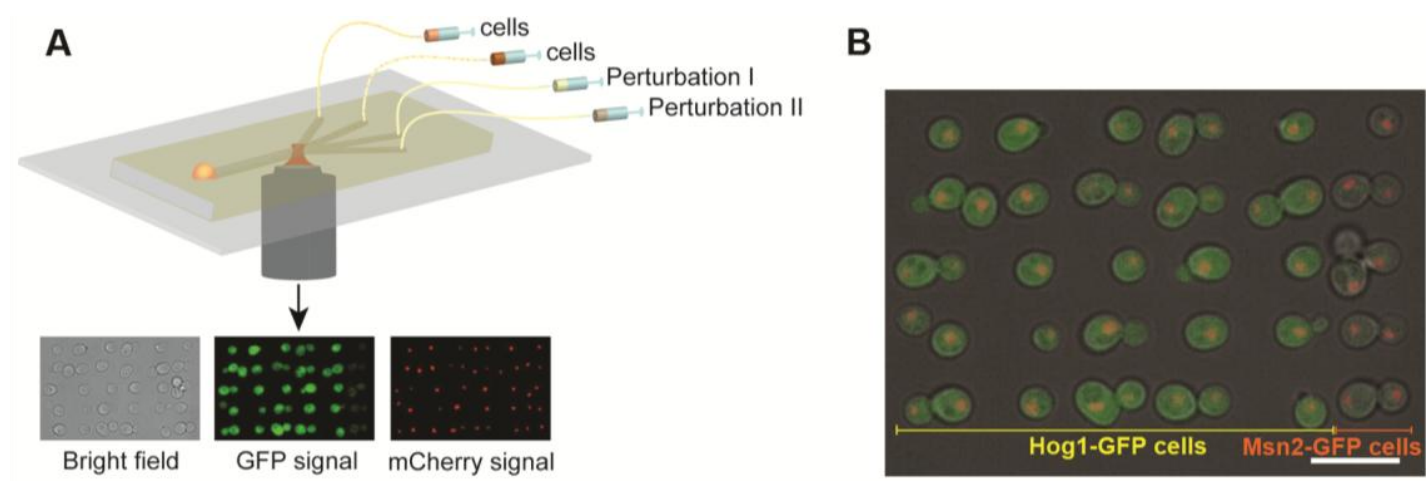

The inner lining of the chamber was initially saturated with the lectin concanavalin A, which promotes cell adhesion between the cell wall sugar residues and the surfaces [26]. Then, four $250 \mu \mathrm{L}$ Hamilton glass syringes (Hamilton Company, Reno, NV, USA) containing the specific cells and different media respectively, were attached to the microfluidic chamber via polytetrafluoroethylene tubing [24].

\subsection{Forming a Two-Type Cell Array Using Optical Tweezers}

Using the $1070 \mathrm{~nm}$ OT (infrared light), single cells were individually chosen; captured and automatically moved from the inlet flow to the cell array site, see Figure 3. Cells are trapped in the focus of the optical trap, $6 \mu \mathrm{m}$ from the bottom surface. The microscope stage is then automatically 
elevated $13 \mu \mathrm{m}$ in order for the bottom surface of the chamber to come in close contact with the cell surface. This assures both small and large yeast cells adhering to the concanavalin A treated glass surface. If the cell is large, the cell is slightly moved out from the focus of the OT even more than a smaller cell, but the forces acting upon it by the microscope stage is neither more or less for differently sized cells. By automating this action for repetition, an array of cells was formed on the chamber floor. The trapping laser exposes each cell to the infrared light only for a few seconds. Experiments confirming that yeast cell viability stays unaffected by this short exposure to the $1070 \mathrm{~nm}$ OT have been performed for short time exposure $(t \leq 10 \mathrm{~s})$ [7].

Figure 3. Shows the channel junction and the cell array site. The inlets are for a: Cells of interest (Hog1-GFP cells), b: Control cells (Msn2-GFP cells), c: Chemical agent one (Hog1-inhibitor), d: Chemical agent one and agent two (sorbitol). The coordinate system $(x, y, z)$ of the microfluidic chamber has its origin at the junction between the two middle inlet channels (marked with + ). The array is placed with the upper most left cell at $(150,-14,0)$. Cells are not drawn to scale.

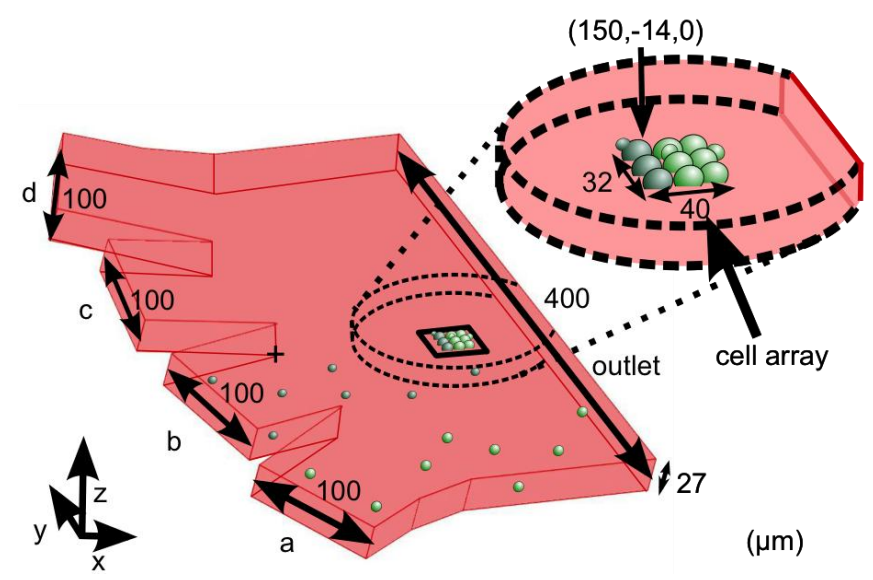

The cell array consisted of a 5-by-5 matrix for the Hog1-GFP single or budding cells and an extra column for the Msn2-GFP control cells, see Figure 2B. Depending on the number of currently budding cells, the cell count in each experiment differs. First, approximately five Msn2-GFP cells were captured in the control cell channel (b) in Figure 3, and positioned in the cell array using the OT. Finally, approximately $25 \mathrm{Hog} 1$-GFP cells were positioned in the same array. Figure 2B shows the cell array after $20 \mathrm{~min}$ of inhibitor treatment but just before sorbitol treatment $(t=-30 \mathrm{~s})$. The cells in the rightmost column are the Msn2-GFP control cells. During imaging, most cells move slightly (hence, the need for a cell tracing software) and occasionally, some cells might even be lost depending on differences in adhesion strength due to presence and/or size of any buds.

\subsection{The Use of Control Cells within the Cell Array}

An issue that had to be resolved when investigating inhibitory events was the fact that a complete inhibition followed by stress treatment will cause the exact same response as a situation where the cells are not exposed to any stress. In order to identify that the array is indeed treated with sorbitol, a column of control cells expressing Msn2-GFP can in our setup be added to the cell array when a complete Hog1 nuclear localization is anticipated. Msn2 is a transcription factor that regulates the 
yeast general stress responses and is regulated by several pathways [27-29]. Msn2 activation is thus induced by several different stresses of which one is high osmolarity. Msn2 translocates from the cytosol to the nucleus upon activation, just like Hog1, where it together with its related transcription factor, Msn4, regulates the expression of $\sim 200$ genes [27,30]. Hence, if Hog1 in inhibitor treated cells do not migrate following stress treatment; an Msn2 migration in the control cells ensures that the cells are de facto exposed to the stress agent. Hence, the usage of control cells for signal detection is a unique and useful approach. In this set of experiments their response is only analyzed qualitatively, due to the high complexity of the Msn2 signaling pathway [31].

\subsection{Flow Simulations, Flow Setup and Array Location}

Changing the flow velocities in the inlet channels creates the change in the media flow around the immobilized cells, which is necessary to trigger intracellular protein migration. In this way, the microenvironment could rapidly be changed from neutral media to a media containing Hog1 inhibitor (for inhibition of Hog1), or sorbitol (for inducing osmotic stress) or a mixture thereof. A sorbitol concentration of $500 \mathrm{mM}$ was used for exposing the cells to osmotic shock. This, quite low, sorbitol concentration was chosen since a higher sorbitol concentration could complicate image analysis due to large changes in cell volume. The automation software OpenLab (PerkinElmer, Waltham, MA, USA) enabled a completely automated setup and maximized the control of the experimental process. OpenLab was used for controlling the mechanical syringe pumps (CMA Microdialysis, Kista, Sweden), the microscope and the EM-CCD camera (C9100-12, Hamamatsu Photonics, Shizuoka, Japan).

The position of the cell array was chosen after performing flow simulations using COMSOL Multiphysics; Chemical Engineering module with application modes "Incompressible Navier-Stokes" and "Convection-Diffusion". The simulations were also used for determining the concentration distributions of the sorbitol and the inhibitor respectively, as well as the velocity field within the device. Computer simulations of the flow profiles and concentration gradients were essential for deciding the location of the cell array. The concentration gradients depend on the flow rates as well as on the diffusion coefficients of sorbitol and the inhibitor respectively. The main advantages with using sorbitol over the commonly used stress agent $\mathrm{NaCl}$ (mean diffusion coefficient $1600 \mu \mathrm{m}^{2} / \mathrm{s}$ [32]) is that sorbitol will provide narrower concentration gradients due to the lower diffusion coefficient and hence lower flow speeds can be used. This is beneficial, since higher flow speeds are more likely to flush the adhered cells away. Another advantage with using sorbitol over $\mathrm{NaCl}$ is that it is not taken up by the cells [33], and does therefore not contribute to the quenching of the GFP signal.

The cell array was positioned to be exposed to a specific concentration from only one inlet channel at a time (e.g., culture medium, inhibitor solution or the sorbitol-inhibitor mixture). Simulations of three different flow configurations of sorbitol and Hog1 inhibitor were performed for the four-inlet microfluidic chamber, respectively (Figure 4):

I. The configuration used when trapping cells with the OT and placing them in an array required flow rates of $80 \mathrm{~nL} / \mathrm{min}$ in the inlet channels containing cells and control cells respectively, and $40 \mathrm{~nL} / \mathrm{min}$ in the inlet channels containing inhibitor and stress.

II. The configuration for inhibitor incubation required flow rates of $40 \mathrm{~nL} / \mathrm{min}$ in the cell, control cell and stress channel respectively and $400 \mathrm{~nL} / \mathrm{min}$ in the inhibitor channel. 
III. The configuration for stress treatment required flow rates of $40 \mathrm{~nL} / \mathrm{min}$ in all inlet channels except the stress channel, which required $800 \mathrm{~nL} / \mathrm{min}$.

In order to have strictly laminar and stable flows at all time points, the flow in any inlet channel was kept to a minimum of $40 \mathrm{~nL} / \mathrm{min}$. According to simulations, the flow velocities at the site of the cell array ( $5 \mu \mathrm{m}$ above the adhesion surface) are approximately $0.8 \mathrm{~mm} / \mathrm{s}$ during inhibitor treatment and $1 \mathrm{~mm} / \mathrm{s}$ during stress treatment. Gustavsson et al. [34] confirmed that sustained glycolytic oscillations in yeast cells stayed unaffected by this $0.2 \mathrm{~mm} / \mathrm{s}$ increase in flow velocity at the cell site. Our measurements confirm that neither the Hog1 response is affected, see Figure 5. A complete detachment of the cell array will occur if increasing the flow rate ten times $(8000 \mathrm{~nL} / \mathrm{min}$ in the stress channel).

Figure 4. Simulation data on concentrations and flow velocities in the microfluidic chamber are shown. The arrow lengths are proportional to the flow velocities given at half of the channel height (a distance of $13.5 \mu \mathrm{m}$ from the bottom surface). The concentration distribution is given at a distance of $0.1 \mu \mathrm{m}$ from the bottom surface. The green area represents the position of the yeast cells and the pump rates are shown in $\mathrm{nL} / \mathrm{min}$ for each inlet channel. The coloring represents the sorbitol flow from 0 to $500 \mathrm{mM}$ (upper row) and the Hog 1 inhibitor flow from 0 to $10 \mu \mathrm{M}$ (lower row) respectively. The first configuration (column I) is used for positioning the cell array using OT, the second configuration (column II) is used for inhibitor incubation and the third configuration (column III) is used for sorbitol treatment and imaging.

I

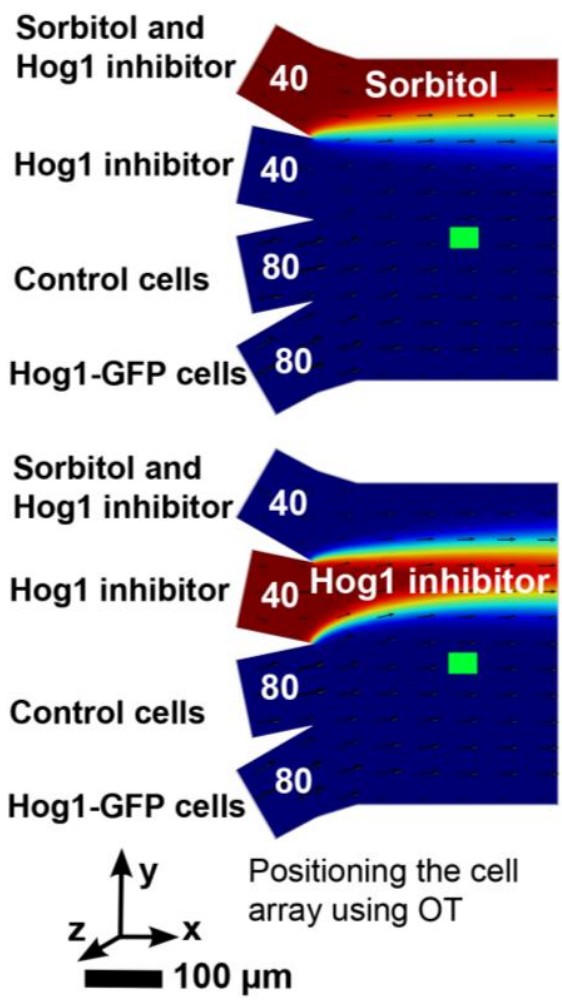

II

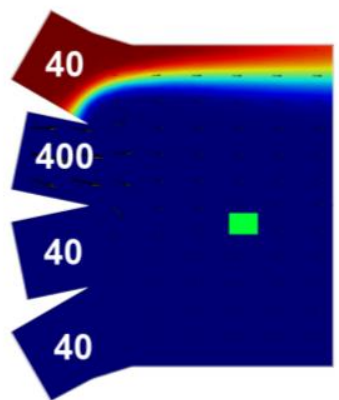

40

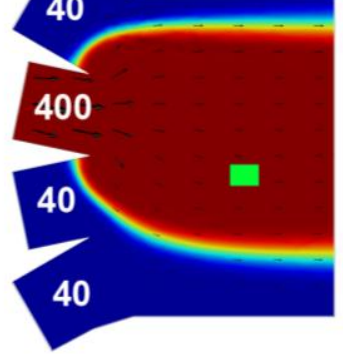

Inhibitor incubation
III

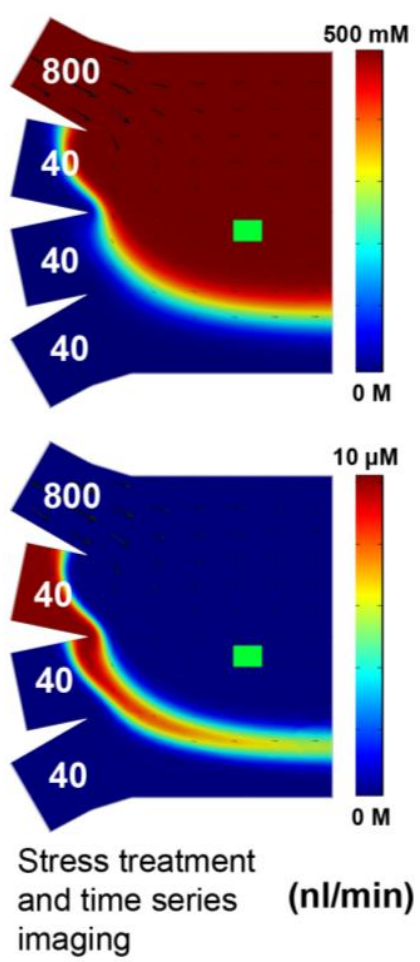


Figure 5. Single-cell response curves for dose-response and inhibition time dependence, respectively. Data were acquired from $t=-30 \mathrm{~s}$ to $t=2700 \mathrm{~s}$ and the sorbitol-inhibitor mixture $(500 \mathrm{mM}$ sorbitol) was introduced into the microfluidic chamber at $t=0 \mathrm{~s}$. (Upper row) Dose-response comparison between (from the left): sorbitol stress only, two different inhibitor concentrations, and the inhibitor only, respectively. The presented data clearly show inhibitor uptake by the cells during $20 \mathrm{~min}$ flow inhibitor exposure. For the experiment with only inhibitor (no sorbitol), the concentration was set to $25 \mu \mathrm{M}$. The figure shows that even a treatment with as low as $5 \mu \mathrm{M}$ Hog1 inhibitor solution induces a partial inhibitory response in the cells. The number of cells in the each plot was from left to right: 86, 52, 51 and 96. (Lower row) Time response comparison of Hog1-GFP nuclear localization due to increased Hog1 inhibitor incubation times from 0 to $20 \mathrm{~min}$. Immediately adding the sorbitol-inhibitor mixture $(500 \mathrm{mM}$ sorbitol) without prior inhibitor incubation, did barely affect the Hog1-GFP nuclear localization, but already after 5 min of inhibitor treatment, a slight decrease of the Hog1-GFP nuclear localization can be seen. The most dramatic decrease in Hog1-GFP nuclear localization could be seen after 10 min of inhibitor treatment compared to the sorbitol only treated cells and the results from the $20 \mathrm{~min}$ incubation time experiments. The number of cells differs depending on the number of budding cells in each experiment, the number of repetitions of the measurements, and was from left to right: 86, 30, 36, 43, 51 and 96.
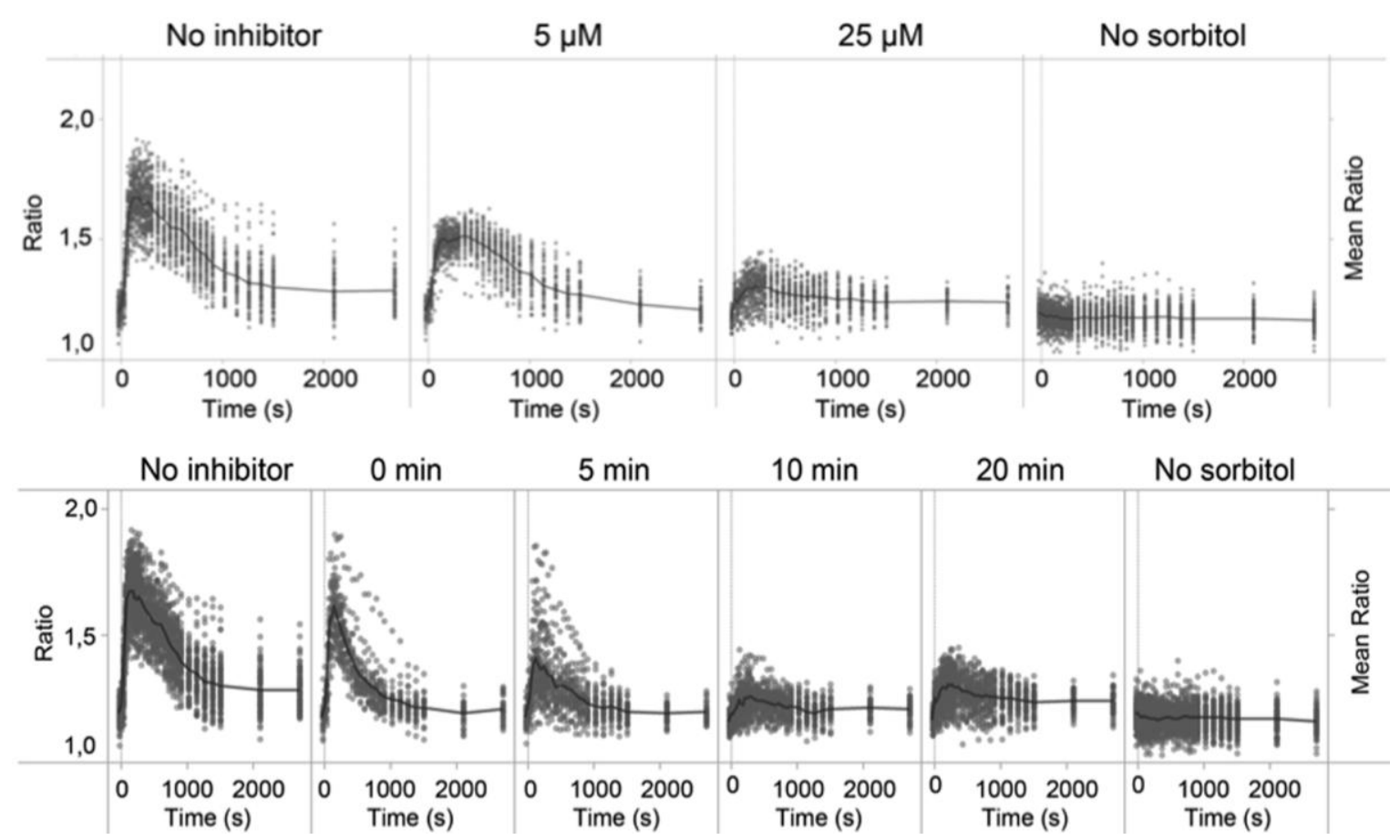

One of the main advantages with the setup is that this single-cell analysis method has a temporal resolution superior to traditional techniques. Additional fluorescein measurements revealed that within only four seconds after pump speed change, the whole cell array is covered with the new intended microenvironment. These measurements also showed that there is no gradient present in the near proximity of the site of the cell array before or after the microenvironment change, confirming the selection of the experimental area as adequate. 


\subsection{Hog I Inhibitor Incubation}

The Hog 1 inhibitor incubation times were varied between 0 and $20 \mathrm{~min}$. The time-lapse imaging started after the inhibitor incubation, at $t=-30 \mathrm{~s}$ and a sorbitol-inhibitor mixture was introduced at $t=0 \mathrm{~s}$. Using a sorbitol-inhibitor mixture instead of pure sorbitol induces osmotic pressure without removing the inhibitory signal. The inhibitor incubation time could be controlled down to a few seconds, as opposed to the previously developed three-inlet microfluidic chamber [7], where the cells had to be pre-treated with the Hog1 inhibitor prior to being introduced to the microfluidic chamber. The temporal control of the inhibitor pre-treatment in this previous chamber was very poor and the mean pre-incubation time was $29 \pm 13$ min (see Supplementary Information, Section S2, Figures S2-S4). In this approach, incubations times ranging from 0 to $20 \mathrm{~min}$ were investigated.

\subsection{Imaging and Analysis}

Exposures were taken every $30 \mathrm{~s}$ for $5 \mathrm{~min}$; every minute for $10 \mathrm{~min}$, every other minute for yet another $10 \mathrm{~min}$ and every tenth minute for $20 \mathrm{~min}$. In order to capture the Hog 1 dynamics while minimizing the bleaching of the fluorophores, the exposures were less frequent as the measurement progressed. The acquiring of the images was performed using the highest level of the attenuator of the EL6000 mercury metal halide bulb, in order for the fluorescent intensity results to be comparable with earlier published single-cell measurements [7]. In order to reduce the photo-bleaching rate, the intensity of the excitation light was kept to a minimum throughout the time-lapse imaging. However, when Hog1-GFP is concentrated to the nucleus, the total bleaching is likely to be faster than when the reporter protein is distributed in the cytosol [35]. The control cells are clearly distinguishable from the Hog1-GFP cells during analysis, by their location and GFP intensity.

For each time point during the time-lapse imaging, an axial stack of seven optical sections with an internal distance of $0.8 \mu \mathrm{m}$ was acquired for bright field, GFP and mCherry respectively [24]. Every image sequence contained 21 photos $(3 \times 7$ photos $)$ per time point, and the maximum imaging frequency was two image sequences per minute. In total, 609 images were analyzed for each experiment. The fluorescence images show the Hog1-GFP/Msn2-GFP and Nrd1-mCherry (a fluorescently marked protein restricted to the nucleus) intensities in every time point. In order to interpret the migration of Hog1-GFP, the exact locations of the cell wall and the nuclear membrane had to be known. This information was retrieved from the outline of the cell contours from a bright field image and the contours of the Nrd1-mCherry intensity, respectively.

The GFP fluorescence intensity was used as a measure of the number of Hog1 proteins in a subcellular location. The images were analyzed using CellStress, an open source image-analysis software for single-cell analysis developed by Smedh et al. [36], and Cellstat. The software automatically identifies optical section where the nucleus is in focus (focal plane), for each individual cell. The mean GFP fluorescence intensity of the cytosol for this particular section is automatically calculated. CellStress uses both the GFP fluorescence images and the bright field images for calculating the ratio $(R)$ between the intensity of the cytosol of the cell focal plane and the nuclear subsection of the cocal plane. The fraction $\mathrm{R}$ is therefore used as an indicator of protein migration. 


$$
R=\frac{\text { Mean intensity in the nuclear area }}{\text { Mean intensity in the cytosol area }} \propto \frac{[\mathrm{Hog} 1 \text { in cuclear area }]}{[\mathrm{Hog} 1 \text { in cytosol area }]}
$$

\section{Results and Discussion}

Yeast cells were captured using OT and were sequentially treated with a selective Hog1 kinase inhibitor and sorbitol. As a result, Hog1-GFP nuclear migration upon sorbitol treatment was impaired post inhibitor exposure. Cellular responses were monitored on a single-cell level by fluorescence microscopy. The results were supported by the use of control cells providing evidence of a congenial setup. A video of the fluorescent response can be found as part of the Supplementary Information (Section S3, Figure S5).

The problem of pre-treatment outside of the microfluidic chamber is now eliminated as a consequence of the introduction of the fourth inlet. The second inlet is still kept neutral, but is now also used for the introduction of the control cells.

\subsection{Constant Flow Situation: Hog1 Inhibitor Treatment of the Adhered Yeast Cell Array}

It was earlier shown that the Hog1 inhibitor could be taken up from a constant flow [24]. However, those measurements were performed without the presence of any control cells. Our following measurements, with control cells, confirm the conclusion that the inhibitor can be taken up from a constant flow. The inhibitor can also be taken up from a stationary environment, where a concentration of $25 \mu \mathrm{M}$ seemed to be the optimal concentration, and the highest concentration where precipitation did not occur (see Supplementary Information, Section S2, Figures S3-S4). Our measurements conclude that an incubation time of ten minutes is the most efficient for maximizing the Hog1 inhibition.

\subsubsection{Dose Response}

Single-cell experiments of an adhered cell array were performed as explained in Section 2. The single-cell data in Figure 5 clearly illustrates the dose dependency of the inhibitory response of the cells. The figure also shows that even a treatment with as low as $5 \mu \mathrm{M}$ Hog 1 inhibitor solution induces a partial inhibitory response in the cells.

Altogether, the nuclear migration of Hog 1 is dramatically decreased and inhibited by approximately $75 \%$ (from the mean ratio values at $t=150 \mathrm{~s}$ ) by a $25 \mu \mathrm{M}$ concentrate of the Hog1 inhibitor 4-(1-benzyl-4-phenyl-1H-1,2,3-triazol-5-yl)- $N$-isopropylpyridin-2-amine, when administrated in a flow in the four-inlet microfluidic chamber. This is in agreement with previous results obtained with the Hog1 inhibitor, from experiments performed on in vitro kinase assays and a yeast cell biofilm [22]. In the aforementioned study, the $\mathrm{IC}_{50}$-value was established to $7.4 \pm 0.41 \mathrm{nM}$. Using whole cells in the microfluidic chamber, our determined $\mathrm{IC}_{50}$-value was $180 \mathrm{nM}$.

Experiments performed using a three-channel system with inhibitor pre-incubation of the cells outside of the system are shown in Supplementary Information, Section S2. A few differences between the cell responses can be seen. Plausible explanations to the differences in inhibition/cellular uptake and initial stress level are the very poor time resolution for the pre-incubation step needed in the three-channel microfluidic chamber, or the difference in flow rates when administering the stress. The 
difference in cellular uptake of the inhibitor could also be caused by one of, or a combination of two things: The flow with which the inhibitor is administrated to the cells in the microfluidic chamber $(400 \mathrm{~nL} / \mathrm{min})$, or the fact that not all of the cell surface is exposed to the inhibitor due to the cells being adhered to the bottom surface. However, it is possible to partly counteract this effect by increasing the inhibitor concentration within the range of solubility of the inhibitor.

\subsubsection{Time Response}

To investigate if the Hog1-GFP nuclear localization response would decrease even further as a function of a shorter inhibitor incubation time, experiments were performed where the inhibitor $(25 \mu \mathrm{M})$ was administrated to the cells $0,5,10$ and $20 \mathrm{~min}$ respectively, before the sorbitol-inhibitor mixture was introduced (Figure 5): Immediately adding the sorbitol-inhibitor mixture without prior inhibitor incubation, did barely affect the amplitude of the mean intensity ratio curve of Hog1-GFP nuclear localization, but a slightly heterogeneous response can be seen here and for 5 min incubation. Already after 5 min of inhibitor treatment, a slight decrease of the Hog1-GFP nuclear localization could be seen. However, the most dramatic decrease in Hog1-GFP nuclear localization (86\% inhibition) could be seen after $10 \mathrm{~min}$ of inhibitor treatment compared to the sorbitol only treated cells. A plausible explanation to this might be that inhibitor molecules taken up by the cell could for instance be rapidly exported by the members of the $\mathrm{ABC}$ transporter superfamily [37,38]. The Hog 1 inhibitor treatment could in future studies be accompanied with $\mathrm{ABC}$ transporter inhibitor treatment (or use mutant yeast cells deficient in export protein/proteins [22]) to facilitate the intracellular residence time of the kinase inhibitor. No correlation between the location of the individual cells in the array and the cell response has been detected.

\section{Conclusions}

In this article, a single-cell study of a selective Hog1 kinase inhibitor [22] was presented using a four-channel microfluidic system, enabling multiple signal inputs (i.e., the Hog1 kinase inhibitor and sorbitol) to a yeast signal transduction pathway and for studying the subsequent dynamic single-cell responses during time-lapse imaging. The presence or absence of a cellular response-activation of the HOG signaling pathway - was monitored by imaging the nuclear translocation of the cytosolic MAPK, Hog1 (fluorescently tagged with GFP) on a single-cell level. This single-cell setup enables cells to be individually traced during the full time period of the experiment. The fourth channel does not only enable controlled, multiple sequential treatments of the cells, it also facilitates a controlled introduction of two different cell strains (e.g., control cells, a mutant or cells treated differently), side by side within the same cell array. Multiple cell strains within the same experiment is a unique feature which is a necessity for, as in this case, determining an expected absent cellular response. The control cells within each experiment had their reporter proteins localized to the nuclei after the equivalent treatment, since the signaling pathway of that reporter protein is not affected by the inhibitor. Hence, the inclusion of these cells further validated the appropriateness of the setup. The data obtained in four-inlet microfluidic chambers are also validated by comparison to results reported in the literature. Hence, the experimental setup of a four-inlet microfluidic chamber, which allows switching between 
two treatments, greatly improves the temporal resolution compared to a system where switching between different perturbation agents is not possible [7].

The main biological conclusions are that a Hog1 inhibitor concentration of $25 \mu \mathrm{M}$ impaired the nuclear Hog1-GFP localization for treated cells with a maximum effect after ten minutes of treatment (86\% inhibition). We have shown that our achieved temporal resolution in the order of seconds was essential in order to determine the most efficient incubation time for $\mathrm{Hog} 1$ inhibition. The $\mathrm{IC}_{50}$-value was established to $180 \mathrm{nM}$, in comparison to the earlier in vitro determined value of $7.4 \pm 0.41 \mathrm{nM}$ [22]. In this case, due to single-cell measurements, we can conclude that the Hog 1 response is mostly homogenous. In addition, kinase inhibition using our approach is a viable alternative to genetic mutation methods when analyzing cellular pathways, circumventing compensatory mechanisms.

Our setup enables a temporal resolution controlled on the level of seconds, with a microscopy resolution on a sub cellular basis. A temporal resolution in this range proved extremely useful for determining the optimal inhibitor treatment time (of a fixed concentration) to be decided. The automatic setup enables very low inter-experimental variation and the custom made automations will provide the opportunities to elaborate with a removal of either perturbation during measurement. This would be extremely hard—not to say impossible — to accomplish using traditional biological methods.

\section{Acknowledgments}

We would like to thank Fraunhofer Chalmers Research Centre Industrial Mathematics (Gothenburg, Sweden) for providing the Cellstat software, and Stefan Hohmann and Peter Dahl at the department of Cell and Molecular Biology (Gothenburg, Sweden) for developing and providing the yeast strains. We would also like to thank the Swedish NMR center (Gothenburg, Sweden) for the diffusion coefficient measurements and Martin Adiels for Tableau support.

This work was supported by the Swedish Research Council (VR), Carl Trygger Foundation for Scientific Research and the European Commission programs AMPKIN and UNICELLSYS. The microfluidic devices were developed at the research facility Centre for Biophysical Imaging, sponsored by the University of Gothenburg.

\section{Conflicts of Interest}

The authors declare no conflict of interest.

\section{References}

1. Ephrussi, B.; Hottinguer, H. Direct demonstration of the mutagenic action of euflavine on baker's yeast. Nature 1950, 166, 956-956.

2. Lindstrom, S.; Andersson-Svahn, H. Overview of single-cell analyses: Microdevices and applications. Lab Chip 2010, 10, 3363-3372.

3. Svahn, H.A.; van den Berg, A. Single cells or large populations? Lab Chip 2007, 7, 544-546.

4. Di Carlo, D.; Lee, L.P. Dynamic single-cell analysis for quantitative biology. Anal. Chem. 2006, $78,7918-7925$. 
5. Raser, J.M.; O'Shea, E.K. Control of stochasticity in eukaryotic gene expression. Science 2004, 304, 1811-1814.

6. Ashkin, A.; Dziedzic, J.M.; Yamane, T. Optical trapping and manipulation of single cells using infrared-laser beams. Nature 1987, 330, 769-771.

7. Eriksson, E.; Sott, K.; Lundqvist, F.; Sveningsson, M.; Scrimgeour, J.; Hanstorp, D.; Goksor, M.; Graneli, A. A microfluidic device for reversible environmental changes around single cells using optical tweezers for cell selection and positioning. Lab Chip 2010, 10, 617-625.

8. Mell, J.C.; Burgess, S.M. Yeast as a Model Genetic Organism. In eLS; John Wiley \& Sons, Ltd.: Hoboken, NJ, USA, 2001.

9. Botstein, D.; Chervitz, S.A.; Cherry, J.M. Genetics-Yeast as a model organism. Science 1997, 277, 1259-1260.

10. Mager, W.H.; Winderickx, J. Yeast as a model for medical and medicinal research. Trends Pharmacol. Sci. 2005, 26, 265-273.

11. Hartwell, L.H. Yeast and cancer. Biosci. Rep. 2004, 24, 523-544.

12. De Nadal, E.; Real, F.X.; Posas, F. Mucins, osmosensors in eukaryotic cells? Trends Cell Biol. 2007, 17, 571-574.

13. Westfall, P.J.; Ballon, D.R.; Thorner, J. When the stress of your environment makes you go HOG wild. Science 2004, 306, 1511-1512.

14. Rep, M.; Krantz, M.; Thevelein, J.M.; Hohmann, S. The transcriptional response of Saccharomyces cerevisiae to osmotic shock-Hot1p and Msn2p/Msn4p are required for the induction of subsets of high osmolarity glycerol pathway-dependent genes. J. Biol. Chem. 2000, 275, 8290-8300.

15. Rep, M.; Reiser, V.; Gartner, U.; Thevelein, J.M.; Hohmann, S.; Ammerer, G.; Ruis, H. Osmotic stress-induced gene expression in Saccharomyces cerevisiae requires Msn1p and the novel nuclear factor Hot1p. Mol. Cell. Biol. 1999, 19, 5474-5485.

16. Thorsen, M.; Di, Y.; Tangemo, C.; Morillas, M.; Ahmadpour, D.; van der Does, C.; Wagner, A.; Johansson, E.; Boman, J.; Posas, F.; et al. The MAPK Hog1p modulates Fps1p-dependent arsenite uptake and tolerance in yeast. Mol. Biol. Cell 2006, 17, 4400-4410.

17. Dihazi, H.; Kessler, R.; Eschrich, K. High osmolarity glycerol (HOG) pathway-induced phosphorylation and activation of 6-phosphofructo-2-kinase are essential for glycerol accumulation and yeast cell proliferation under hyperosmotic stress. J. Biol. Chem. 2004, 279, 23961-23968.

18. Posas, F.; WurglerMurphy, S.M.; Maeda, T.; Witten, E.A.; Thai, T.C.; Saito, H. Yeast HOG1 MAP kinase cascade is regulated by a multistep phosphorelay mechanism in the SLN1-YPD1-SSK1 "two-component” osmosensor. Cell 1996, 86, 865-875.

19. Tatebayashi, K.; Tanaka, K.; Yang, H.-Y.; Yamamoto, K.; Matsushita, Y.; Tomida, T.; Imai, M.; Saito, H. Transmembrane mucins Hkr1 and Msb2 are putative osmosensors in the SHO1 branch of yeast HOG pathway. EMBO J. 2007, 26, 3521-3533.

20. Klipp, E.; Nordlander, B.; Kruger, R.; Gennemark, P.; Hohmann, S. Integrative model of the response of yeast to osmotic shock. Nat. Biotechnol. 2005, 23, 975-982. 
21. Ferrigno, P.; Posas, F.; Koepp, D.; Saito, H.; Silver, P.A. Regulated nucleo/cytoplasmic exchange of HOG1 MAPK requires the importin beta homologs NMD5 and XPO1. EMBO J. 1998, 17, 5606-5614.

22. Dinér, P.; Veide Vilg, J.; Kjellén, J.; Migdal, I.; Andersson, T.; Gebbia, M.; Giaever, G.; Nislow, C.; Hohmann, S.; Wysocki, R.; et al. Design, synthesis, and characterization of a highly effective Hog1 inhibitor: A powerful tool for analyzing MAP kinase signaling in yeast. PLoS One 2011, 6, e20012; doi:10.1371/journal.pone.0020012.

23. Sott, K.; Eriksson, E.; Goksör, M. Biomolecular Separation and Analysis. In Lab-on-a-Chip Technology; Herold, K., Rasooly, A., Eds.; Caister Academic Press: Norwich, UK, 2009.

24. Hamngren, C.; Diner, P.; Grotli, M.; Goksor, M.; Adiels, C.B. Design and Evaluation of a Microfluidic System for Inhibition Studies of Yeast Cell Signaling. In Proceedings of Optical Trapping and Optical Micromanipulation IX, San Diego, CA, USA, 12 August 2012; Dholakia, K., Spalding, G.C., Eds.; SPIE: San Diego, CA, USA, 2012; Volume 8458.

25. Duffy, D.C.; McDonald, J.C.; Schueller, O.J.A.; Whitesides, G.M. Rapid prototyping of microfluidic systems in poly(dimethylsiloxane). Anal. Chem. 1998, 70, 4974-4984.

26. Mislovicova, D.; Masarova, J.; Svitel, J.; Gemeiner, P. Influence of mannan epitopes in glycoproteins-Concanavalin A interaction. Comparison of natural and synthetic glycosylated proteins. Int. J. Biol. Macromol. 2002, 30, 251-258.

27. Gorner, W.; Durchschlag, E.; Martinez-Pastor, M.T.; Estruch, F.; Ammerer, G.; Hamilton, B.; Ruis, H.; Schuller, C. Nuclear localization of the $\mathrm{C} 2 \mathrm{H} 2$ zinc finger protein Msn2p is regulated by stress and protein kinase A activity. Gene Dev. 1998, 12, 586-597.

28. Gorner, W.; Durchschlag, E.; Wolf, J.; Brown, E.L.; Ammerer, G.; Ruis, H.; Schuller, C. Acute glucose starvation activates the nuclear localization signal of a stress-specific yeast transcription factor. ЕMBO J. 2002, 21, 135-144.

29. Hirata, Y.; Andoh, T.; Asahara, T.; Kikuchi, A. Yeast glycogen synthase kinase-3 activates Msn2p-dependent transcription of stress responsive genes. Mol. Biol. Cell 2003, 14, 302-312.

30. MartinezPastor, M.T.; Marchler, G.; Schuller, C.; MarchlerBauer, A.; Ruis, H.; Estruch, F. The Saccharomyces cerevisiae zinc finger proteins Msn2p and Msn4p are required for transcriptional induction through the stress-response element (STRE). EMBO J. 1996, 15, 2227-2235.

31. Petrenko, N.; Chereji, R.V.; McClean, M.N.; Morozov, A.V.; Broach, J.R. Noise and interlocking signaling pathways promote distinct transcription factor dynamics in response to different stresses. Mol. Biol. Cell 2013, 24, 2045-2057.

32. Vanýsek, P. CRC Handbook of Chemistry and Physics; Lide, D.R., Ed.; CRC Press: Boca Raton, FL, USA, 2005.

33. Karlgren, S.; Pettersson, N.; Nordlander, B.; Mathai, J.C.; Brodsky, J.L.; Zeidel, M.L.; Bill, R.M.; Hohmann, S. Conditional osmotic stress in yeast-A system to study transport through aquaglyceroporins and osmostress signaling. J. Biol. Chem. 2005, 280, 7186-7193.

34. Gustavsson, A.-K.; Adiels, C.B.; Goksor, M. Induction of Sustained Glycolytic Oscillations in Single Yeast Cells Using Microfluidics and Optical Tweezers. In Proceedings of Optical Trapping and Optical Micromanipulation IX, San Diego, CA, USA, 12 August 2012; Dholakia, K., Spalding, G.C., Eds.; SPIE: San Diego, CA, USA, 2012; Volume 8458. 
35. Frey, S.; Sott, K.; Smedh, M.; Millat, T.; Dahl, P.; Wolkenhauer, O.; Goksor, M. A mathematical analysis of nuclear intensity dynamics for Mig1-GFP under consideration of bleaching effects and background noise in Saccharomyces cerevisiae. Mol. Biosyst. 2011, 7, 215-223.

36. Smedh, M.; Beck, C.; Sott, K.; Goksör, M. CellStress-Open Source Image Analysis Program for Single-Cell Analysis. In Proceedings of Optical Trapping and Optical Micromanipulation VII, San Diego, CA, USA, 1 August 2010; Dholakia, K., Spalding, G.C., Eds.; SPIE: San Diego, CA, USA, 2010; Volume 7762.

37. Decottignies, A.; Goffeau, A. Complete inventory of the yeast ABC proteins. Nat. Genet. 1997, 15, 137-145.

38. Nelissen, B.; DeWachter, R.; Goffeau, A. Classification of all putative permeases and other membrane plurispanners of the major facilitator superfamily encoded by the complete genome of Saccharomyces cerevisiae. FEMS Microbiol. Rev. 1997, 21, 113-134.

(C) 2014 by the authors; licensee MDPI, Basel, Switzerland. This article is an open access article distributed under the terms and conditions of the Creative Commons Attribution license (http://creativecommons.org/licenses/by/3.0/). 